\title{
Contribution of Series Resistance in Modelling of High-Temperature Type II Superlattice p-i-n Photodiodes
}

\author{
Jarosław Wróbel, Piotr Martyniuk, and Antoni Rogalski \\ Institute of Applied Physics, Military University of Technology, 2 Kaliskiego Street, 00-908 Warsaw, Poland \\ Correspondence should be addressed to Jarosław Wróbel, jarekwrobel@wat.edu.pl
}

Received 24 August 2012; Accepted 23 October 2012

Academic Editor: Ovidio Salvetti

Copyright ( $) 2012$ Jarosław Wróbel et al. This is an open access article distributed under the Creative Commons Attribution License, which permits unrestricted use, distribution, and reproduction in any medium, provided the original work is properly cited.

We analyze some of the consequences of omitting series resistance in InAs/GaSb p-i-n T2SL photodiode dark current modelling, using simplified p-n junction model. Our considerations are limited to generation-recombination and diffusion-effective carrier lifetimes to show the possible scale of over- or underestimating photodiodes parameters in high-temperature region. As is shown, incorrect series resistance value might cause discrepancies in $\tau_{\mathrm{gr}}$ and $\tau_{\text {diff }}$ 's estimations over one order of magnitude.

\section{Introduction}

Type II superlattices (T2SLs), especially InAs/GaSb, are very promising materials for both uncooled as well as cooled midwavelength infrared (MWIR) and long wavelength infrared (LWIR) photodetectors [1]. Relative easiness of controlling band offset causes them to be used in different types of structures [2]. Especially, introducing unipolar barriers in various designs based on type II SLs drastically changed the architecture of infrared detectors. At present, the InAs/GaSb T2LSs are considered to be an alternative to the HgCdTe IR material systems [1] and a candidate for the third-generation IR detectors [3]. However, InAs/GaSb T2SL is in an early stage of development. Problems exist in material growth, processing, substrate preparation, and device passivation [46]. Moreover, correct interpretations of measured detector characteristics are often difficult due to fact that T2SLs' band structures are much more complicated than bulk materials [7]. From this reason, there have been developed many simplified models [8-13], which assume that T2SL electrical parameters are mainly dependent on energy difference between first conduction and heavy hole miniband (which is treated as an effective bandgap). For an approximative describing of the detector transport mechanisms, the wellknown standard theory of $\mathrm{p}-\mathrm{n}$ junction is used $[2,14]$. Recently published results of our group show that usage of this method can give very good fitting between theoretical predictions and experimental data in a wide range of bias voltage (from -1.6 to $+0.3 \mathrm{~V}$ ) and temperature (from 77 to $240 \mathrm{~K})$ for $\mathrm{p}-\mathrm{i}-\mathrm{n}$ and $\mathrm{nBn}$ devices [15-17]. It appears that good agreement between both types of results is possible if the influence of series resistance $\left(R_{\text {series }}\right)$ is taken into consideration, what might be essential in a thermoelectrically cooled (TE) photodetectors (in temperature range above $180 \mathrm{~K})$.

In this paper we present the consequences of $R_{\text {series }}$ omitting in the dark current modelling of p-i-n T2SL junctions, often met in literature. These consequences are shown on an example of temperature dependence of two fitting parameters-generation-recombination $\left(\tau_{\mathrm{gr}}\right)$ and diffusion $\left(\tau_{\text {diff }}\right)$-effective carrier lifetimes. Both two mechanisms have decisive influence on current-voltage characteristics of TEcooled p-i-n T2SL junctions in near-zero bias voltages [17].

\section{Experimental Results}

In the studies we have chosen representative sample of 10/10 ML InAs/GaSb T2SL in a p-i-n structure, fabricated in the Center for High Technology Materials, University of New Mexico, Albuquerque, NM, USA. The photodiode cutoff wavelength is roughly equal to $5.6 \mu \mathrm{m}$ at $120 \mathrm{~K}$ and $6.2 \mu \mathrm{m}$ at $230 \mathrm{~K}$ [18]. The detector architecture design and measurement details of current-voltage characteristics of 


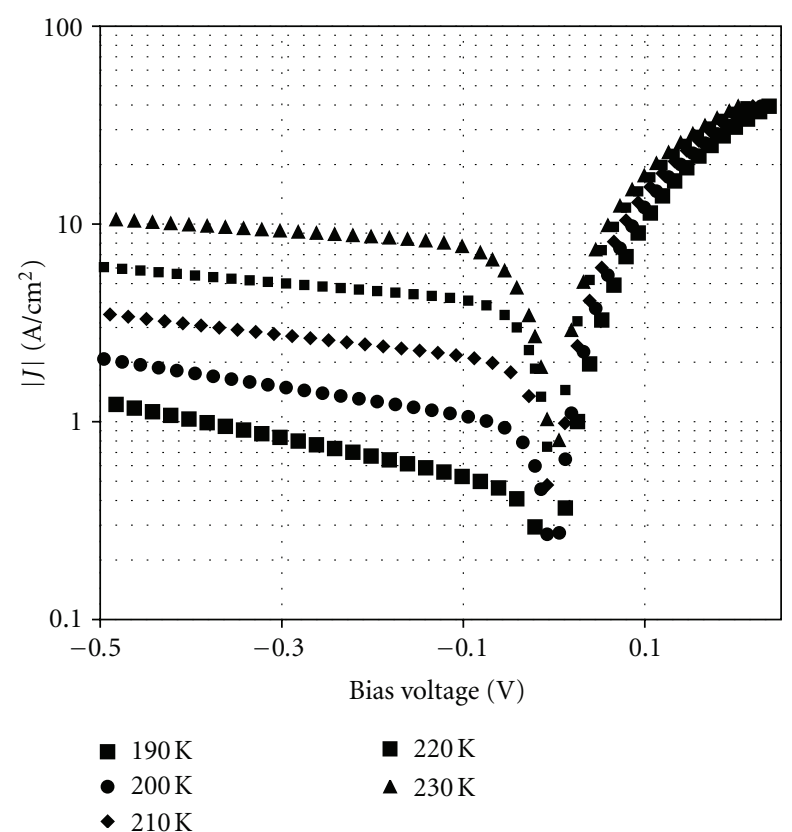

FIGURE 1: Experimental dark current density-voltage characteristics of p-i-n T2SL photodiode at different temperatures (after [17]).

these samples were presented elsewhere [16, 17]. Here we present only a few of the results (see Figures 1 and 2), indispensable for presenting the main goal of the paper.

\section{Modelling of Current-Voltage Characteristics}

In order to explain current-voltage characteristics of the MWIR type-II SLS photodiodes a bulk-based model with an effective band gap of SL material is used. It is well recognized that the photodiode dark current can be found as a superposition of several mechanisms (see Figure 3):

$$
I_{\text {dark }}=I_{\text {diff }}+I_{\mathrm{gr}}+I_{\mathrm{btb}}+I_{\mathrm{tat}}+I_{R \text { shunt }},
$$

including four main mechanisms: diffusion ( $\left.I_{\text {diff }}\right)$, generation-recombination $\left(I_{\mathrm{gr}}\right)$, band-to-band tunnelling $\left(I_{\mathrm{btb}}\right)$, and trap-assisted tunnelling $\left(I_{\mathrm{tat}}\right)$. The remaining mechanism is current due to the shunt resistance $\left(I_{R s h u n t}\right.$, originates from the surface and bulk leakage current and shows the presence in the reverse bias region).

The relation between the applied voltage, $V$, and the diffusion current density, $J_{\text {diff, }}$, is given by

$$
J_{\text {diff }}=J_{s}\left[\exp \left(\frac{q V}{k T}-1\right)\right],
$$

where $q$ is the electron charge, $k$ is the Boltzmann's constant, and $T$ is the absolute temperature. In the case of our devices, due to a gradient doping profile at the contacts, we apply the reflective contact configuration, and then the saturation current density $J_{s}$ can be expressed as

$$
J_{s}=q n_{i}^{2}\left(\frac{D_{e}}{N_{a} L_{e}} \tanh \frac{x_{p}}{L_{e}}+\frac{D_{h}}{N_{d} L_{h}} \tanh \frac{x_{n}}{L_{h}}\right),
$$

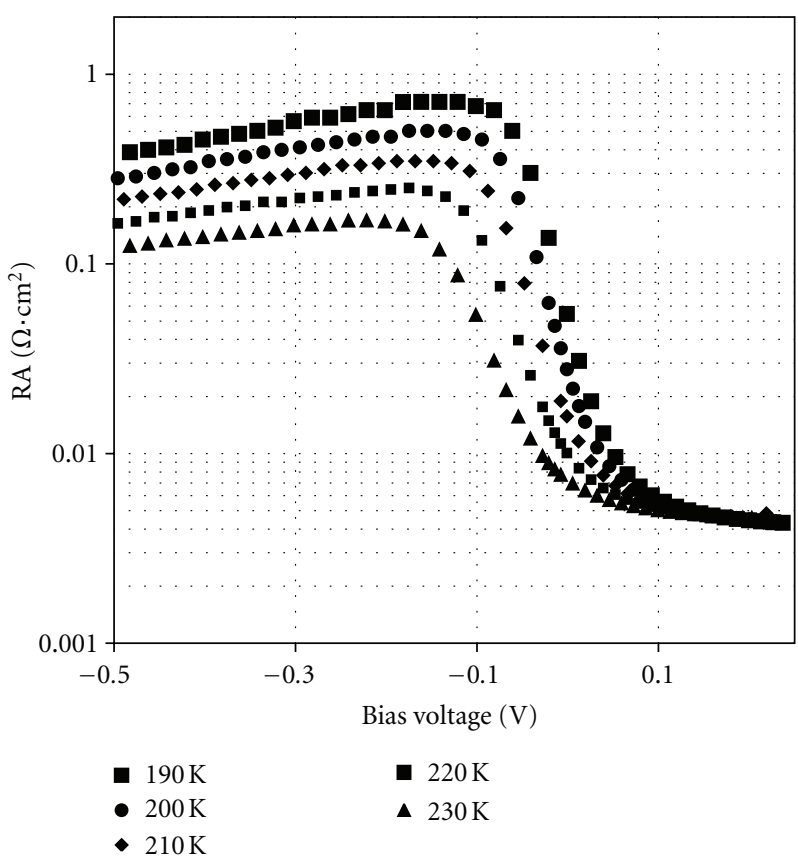

Figure 2: Experimental resistance-area product versus bias voltage of p-i-n T2SL photodiode at different temperatures (after [17]).

where $n_{i}$ is the intrinsic carrier concentration, $N_{a, d}$ is acceptor/donor doping concentrations, $\mu_{e, h}$ is minority electron/hole mobility, and $x_{n, p}$ is the device thicknesses $\left(x_{n}\right.$ is the thickness of $n$-type region, instead $x_{p}$ is the thickness of $p$ type region). Additionally, $L_{e, h}$ means electron/hole diffusion current length, and $D_{e, h}$ diffusivity (with diffusion lifetime $\left.\tau_{e, h}\right)$, where

$$
D_{e, h}=\frac{k T}{q} \mu_{e, h}, \quad L_{e, h}=\sqrt{D_{e, h} \tau_{e, h}} .
$$

The g-r current density under reverse-bias voltage and for forward-bias voltage values, that are less than $V_{b}$ by several $k T / q$, is derived as

$$
J_{\mathrm{gr}}=\frac{q n_{i} w}{\left(\tau_{\mathrm{eo}} \tau_{\mathrm{ho}}\right)^{1 / 2}} \frac{2 \sinh (q V / 2 k T)}{q\left(V_{b}-V\right) / k T} f(b),
$$

where $\tau_{\mathrm{eo}}$ and $\tau_{\mathrm{ho}}$ are the carrier lifetimes for electrons and holes within the depletion region ( $w$ is the depletion width). In our estimation we also assumed that $\tau_{\mathrm{eo}}=\tau_{\mathrm{ho}}=\tau_{\mathrm{gr}}$ and $\tau_{e}=\tau_{h}=\tau_{\text {diff. }}$. The function $f(b)$ is a complicated expression involving a trap level and an applied voltage [2]. The values of parameters used in a device modelling are taken from published literature [19-21] and are gathered in Table 1 [16].

The description of other mechanisms, essential in high reverse bias voltage condition and detailed discussion of fitting procedure (including simple method of avoiding difficulties connected with the influence of series resistance), can be found in [16].

\section{Results and Discussions}

In some of the recently published papers related to high operating temperature (HOT) T2SL devices (see, e.g., Cervera et al. [12]) is mentioned the difficulties connected with 
TABle 1: Parameters taken in modelling of MWIR InAs/GaSb superlattice photodiodes.

\begin{tabular}{lc}
\hline $\begin{array}{l}\text { Device geometry } \\
\text { thickness of } p \text {-type region, } x_{p}\end{array}$ & $0.27 \mu \mathrm{m}$ \\
thickness of $n$-type region, $x_{n}$ & $2.25 \mu \mathrm{m}$ \\
$\quad$ electrical area & $2.03 \times 10^{-3} \mathrm{~cm}^{2}$ \\
Donor concentration, $N_{d}$ & $1 \times 10^{16} \mathrm{~cm}^{-3}$ \\
Acceptor concentration, $N_{a}$ & $1 \times 10^{18} \mathrm{~cm}^{-3}$ \\
Trap concentration, $N_{T}$ & $8 \times 10^{14} \mathrm{~cm}^{-3}$ \\
Minority electron mobility, $\mu_{e}$ & $10000 \mathrm{~cm}^{2} / \mathrm{Vs}$ \\
Minority hole mobility, $\mu_{h}$ & $1000 \mathrm{~cm}^{2} / \mathrm{Vs}$ \\
Electron-effective mass, $m_{e}^{*}$ & $0.015 \mathrm{~m}_{0}$ \\
Hole effective mass, $m_{h}^{*}$ & $0.4 m_{0}$ \\
\hline
\end{tabular}

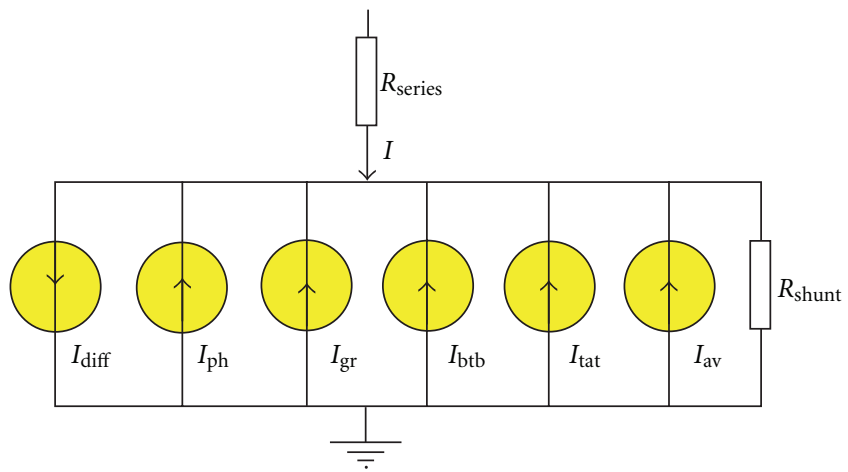

Figure 3: Possible currents operating in the photodiode. $I_{\text {diff }}$ is ideal diffusion current, $I_{\mathrm{ph}}$ is the photocurrent, $I_{\mathrm{gr}}$ is due to a generation-recombination mechanism, $I_{\mathrm{btb}}$ is due to a band-toband tunnelling, $I_{\text {tat }}$ is due to a trap-to-band tunnelling, and $R_{\text {shunt }}$ is due to a surface and bulk leakage shunt resistance. Limiting currents act in opposition to diffusion current.

fitting procedure between measured $I-V$ characteristics and theoretical predicted results above $200 \mathrm{~K}$. In our opinion these difficulties are strictly connected with the influence of series resistance in high-temperature region. Because $R_{\text {series }}{ }^{-}$ value is connected in a series to all generation-recombination mechanisms (see Figure 3), we should solve the nonlinear problem to obtain voltage drops on both whole device except series resistance and a series resistance separately (see discussion in [16]). Omitting this problem causes it to be impossible to fit to measured dynamic resistancevoltage characteristics, $R_{d}(V)$, under HOT conditions. It is caused by the fact that voltage drop on device except $R_{\text {series }}$ might be essentially lower than measured voltage, what causes considerable changes in shapes of RA-product curves (see Figure 4), and, in consequence, great difficulties in theoretical fitting procedure to the experimental $R_{d}(V)$ characteristics.

At the beginning, we present a comparison of two simulation results, which were made using the same input parameters, except $R_{\text {series. }}$. As we can see on Figure 4, the difference in a bias-dependent peak positions of dynamic resistance is nearly equal to $0.1 \mathrm{~V}$, what in consequence causes significance discrepancy in an effective lifetimes estimations from (2) and (5).

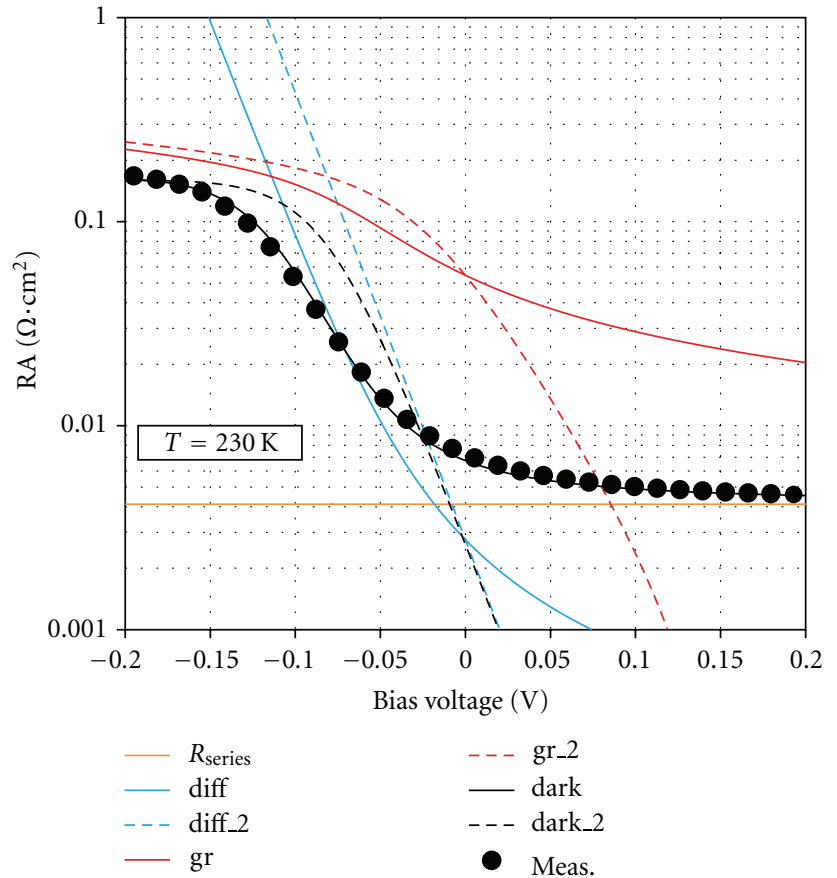

FIgURE 4: The comparison of two simulation results at $230 \mathrm{~K}$, assuming the same input parameters except $R_{\text {series }}$. Curves with 2 -index are calculated assuming zero value of $R_{\text {series }}$. Plots with no additional indexes are calculated assuming that $R_{\text {series }} \approx 2 \Omega$ obtained from the fitting to the measured dynamic resistance (see Figure 2 ) under the forward bias voltage condition (at $\approx 0.2 \mathrm{~V}$ ).

Second aspect, previously mentioned, is the fact that influences of $R_{\text {series }}$ on shapes of different dark current components are effective if the $R_{\text {series }}$ value is comparable with dynamic resistance contributions of respective dark current components. This is especially visible for g-r mechanism. As a result, estimating of $\tau_{\mathrm{gr}}$ and $\tau_{\text {diff }}$ data assuming incorrect $R_{\text {series }}$ value causes significant over- or underestimation in the effective current lifetimes.

Assuming the consideration above, we should underline that it is impossible to fit to measured $\mathrm{RA}(V)$ characteristics using (2) and (5), and omitting $R_{\text {series. }}$. It causes many researchers to try to fit roughly only to the $I-V$ characteristics, without analysing first derivative of it $(\mathrm{RA}(V)$ dependence). In a consequence, for avoiding overestimation of dark current in a near zero-bias voltage region, it is needed to increase $\tau_{\text {diff }}$ and decrease $\tau_{\mathrm{gr}}$ at bias voltage about $-0.2 \mathrm{~V}$ in our case (this value depends on sample, temperature, and $R_{\text {series }}$ ) shown in Figure 5. Curves with no additional index are calculated using parameters obtained from the correct fitting, but with zero value of $R_{\text {series }}$. Plots with 2 indexes are calculated using incorrect procedure.

As we can see on Figure 6, discrepancies between fitting results, made with and without including $R_{\text {series }}$, increase with temperature. It is caused by the fact that ideal photodiode resistance of p-i-n T2SL photodiode (with $R_{\text {series }}=$ 0 ) decreases with temperature and becomes comparable with weak temperature-dependent series resistance at hightemperature operation. In a consequence, a voltage drop 


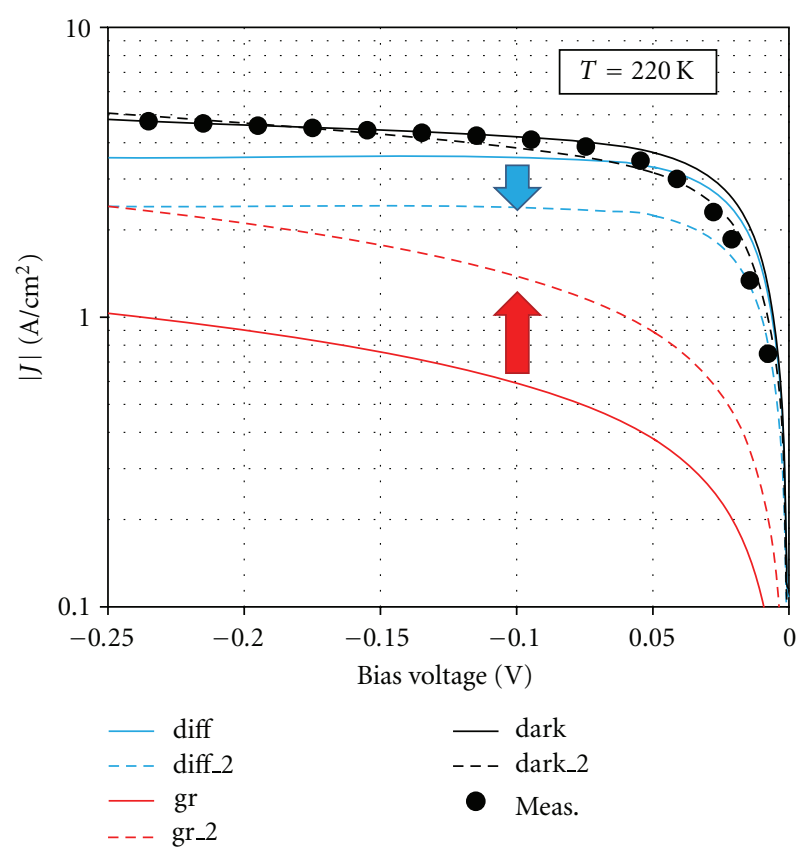

FIGURE 5: Explanation of incorrect fitting procedure (for more details-see text). Arrows show tendencies in change components of dark current to obtain good fitting to experimental data.

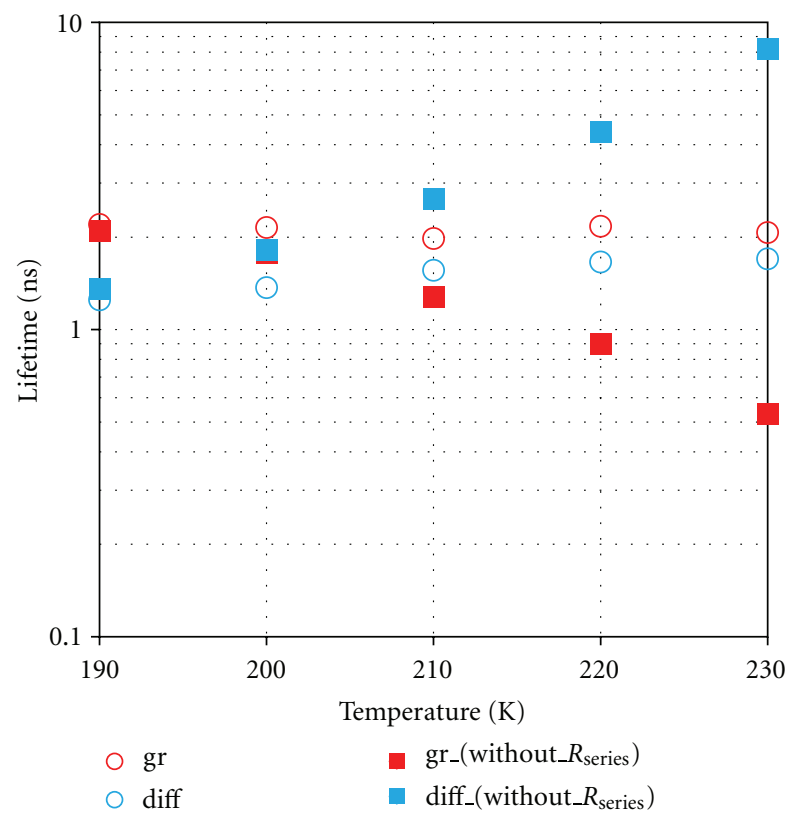

Figure 6: Comparison of g-r and diff. effective current lifetimes estimated theoretically for two cases: with including $R_{\text {series }}$ and without it (see additional description in the text).

on an ideal diode and on series resistance are comparable, and $I-V$ characteristics essentially change their shapes. This results in under- or overestimations in key photodiode fitting parameters, like $\tau_{\mathrm{gr}}$ or $\tau_{\text {diff. }}$ Our results for the MWIR potodiode seem to be confirmed by more direct measurements [22], where effective current lifetimes are weekly temperature dependent over $200 \mathrm{~K}$.

\section{Conclusions}

In this paper we analyze some of the consequences of omitting series resistance in InAs/GaSb p-i-n T2SL photodiode dark current modelling, using simplified p-n junction model. Our considerations are limited to generation-recombination and diffusion-effective carrier lifetimes to show the possible scale of over- or underestimating photodiodes parameters. As is shown, incorrect series resistance value might cause discrepancies between $\tau_{\mathrm{gr}}$ and $\tau_{\text {diff }}$ over one order of magnitude. Our results seem to be good explanation of difficulties in the estimation of parameters of MWIR T2SL photodiodes operated in high-temperature region.

\section{Acknowledgment}

This paper has also been done under financial support of the Polish Ministry of Sciences and Higher Education, Key Project POIG.01.03.01-14-016/08 "New Photonic Materials and their Advanced Application."

\section{References}

[1] A. Rogalski and P. Martyniuk, "InAs/GaInSb superlattices as a promising material system for third generation infrared detectors," Infrared Physics \& Technology, vol. 48, no. 1, pp. 3952, 2006.

[2] A. Rogalski, Infrared Detectors, CRC Press, Boca Raton, Fla, USA, 2nd edition, 2010.

[3] A. Rogalski, "HgCdTe infrared detector material: history, status and outlook," Reports on Progress in Physics, vol. 68, no. 10, pp. 2267-2336, 2005.

[4] H. S. Kim, E. Plis, A. Khoshakhlagh et al., "Performance improvement of InAs/GaSb strained layer superlattice detectors by reducing surface leakage currents with SU-8 passivation," Applied Physics Letters, vol. 96, no. 3, Article ID 033502, 3 pages, 2010.

[5] H. S. Kim, Investigation of InAs/GaSb superlattice based nBn detectors and focal plane arrays [Ph.D. thesis], Optical Science and Engineering, University of New Mexico, 2010.

[6] H. S. Kim, E. Plis, N. Gautam et al., "SU-8 passivation of typeII InAs/GaSb strained layer superlattice detectors," in Infrared Technology and Applications 36, vol. 7660 of Proceedings of SPIE, 76601U-1-9, April 2010.

[7] F. Szmulowicz, H. J. Haugan, G. J. Brown et al., "Interfaces as design tools for short-period InAs/GaSb type-II superlattices for mid-infrared detectors," Opto-Electronics Review, vol. 14, no. 1, pp. 71-77, 2006.

[8] D. R. Rhiger, R. E. Kvaas, S. F. Harris, and C. J. Hill, "Characterization of LWIR diodes on InAs/GaSb type-II superlattice material," Infrared Physics \& Technology, vol. 52, no. 6, pp. 304-309, 2009.

[9] J. Nguyen, D. Z. Ting, C. J. Hill, A. Soibel, S. A. Keo, and S. D. Gunapala, "Dark current analysis of InAs/GaSb superlattices at low temperatures," Infrared Physics \& Technology, vol. 52, no. 6, pp. 317-321, 2009.

[10] C. L. Canedy, E. H. Aifer, J. H. Warner et al., "Controlling dark current in type-II superlattice photodiodes," Infrared Physics \& Technology, vol. 52, no. 6, pp. 326-334, 2009.

[11] I. Vurgaftman, C. L. Canedy, J. A. Nolde, C. A. Affouda et al., "Analysis and performance of type-II superlattice infrared detectors," Optical Engineering, vol. 50, no. 6, Article ID 061007, 2011. 
[12] C. Cervera, K. Jaworowicz, H. Aït-Kaci et al., "Temperature dependence performances of InAs/GaSb superlattice photodiode," Infrared Physics \& Technology, vol. 54, no. 3, pp. 258-262, 2011.

[13] V. Gopal, E. Plis, J. B. Rodriguez, C. E. Jones, L. Faraone, and S. Krishna, "Modeling of electrical characteristics of midwave type II InAs/GaSb strain layer superlattice diodes," Journal of Applied Physics, vol. 104, no. 12, Article ID 124506, 6 pages, 2008.

[14] C.-T. Sah, R. N. Noyce, and W. Shockley, "Carrier generation and recombination in $\mathrm{P}-\mathrm{N}$ junctions and $\mathrm{P}-\mathrm{N}$ junction characteristics," Proceedings of the Institute of Radio Engineers, vol. 45, no. 9, pp. 1228-1243, 1957.

[15] P. Martyniuk, J. Wróbel, E. Plis et al., "Performance modeling of MWIR InAs/GaSb/B- $\mathrm{Al}_{0.2} \mathrm{Ga}_{0.8}$ Sb type-II superlattice $\mathrm{nBn}$ detector," Semiconductor Science and Technology, vol. 27, no. 5, Article ID 055002, 2012.

[16] J. Wróbel, E. Plis, P. Madejczyk et al., "Analysis of temperature dependence of dark current mechanisms for mid-wavelength infrared InAs/GaSb superlatticephotodiodes," submitted.

[17] J. Wróbel, P. Martyniuk, E. Plis et al., "Dark current modeling of MWIR type-II superlattice detectors," in Infrared Technology and Applications 38, vol. 8353 of Proceedings of SPIE, 835316, April 2012.

[18] B. Klein, E. Plis, M. N. Kutty et al., "Varshni parameters for InAs/GaSb strained layer superlattice infrared photodetectors," Journal of Physics D, vol. 44, no. 7, Article ID 075102, 5 pages, 2011.

[19] T. V. Chandrasekhar Rao, J. Antoszewski, J. B. Rodriguez, E. Plis, S. Krishna, and L. Faraone, "Quantitative mobility spectrum analysis of carriers in GaSb/InAs/GaSb superlattice," Journal of Vacuum Science and Technology B, vol. 26, no. 3, pp. 1081-1083, 2008.

[20] F. Szmulowicz, H. J. Haugan, S. Elhamri, G. J. Brown, and W. C. Mitchel, "Transport studies of MBE-grown InAs/GaSb superlattices," Opto-Electronics Review, vol. 18, no. 3, pp. 267270, 2010.

[21] D. Z.-Y. Ting, A. Soibel, L. Höglund et al., "Type-II superlattice infrared detectors," in Semiconductors and Semimetals, S. D. Gunapala, D. R. Rhiger, and C. Jagadish, Eds., vol. 84, pp. 157, Elsevier, Amsterdam, The Netherlands, 2011.

[22] E. C. F. da Silva, D. Hoffman, A. Hood, B. M. Nguyen, P. Y. Delaunay, and M. Razeghi, "Influence of residual impurity background on the nonradiative recombination processes in high purity InAs/GaSb superlattice photodiodes," Applied Physics Letters, vol. 89, no. 24, Article ID 243517, 3 pages, 2006. 

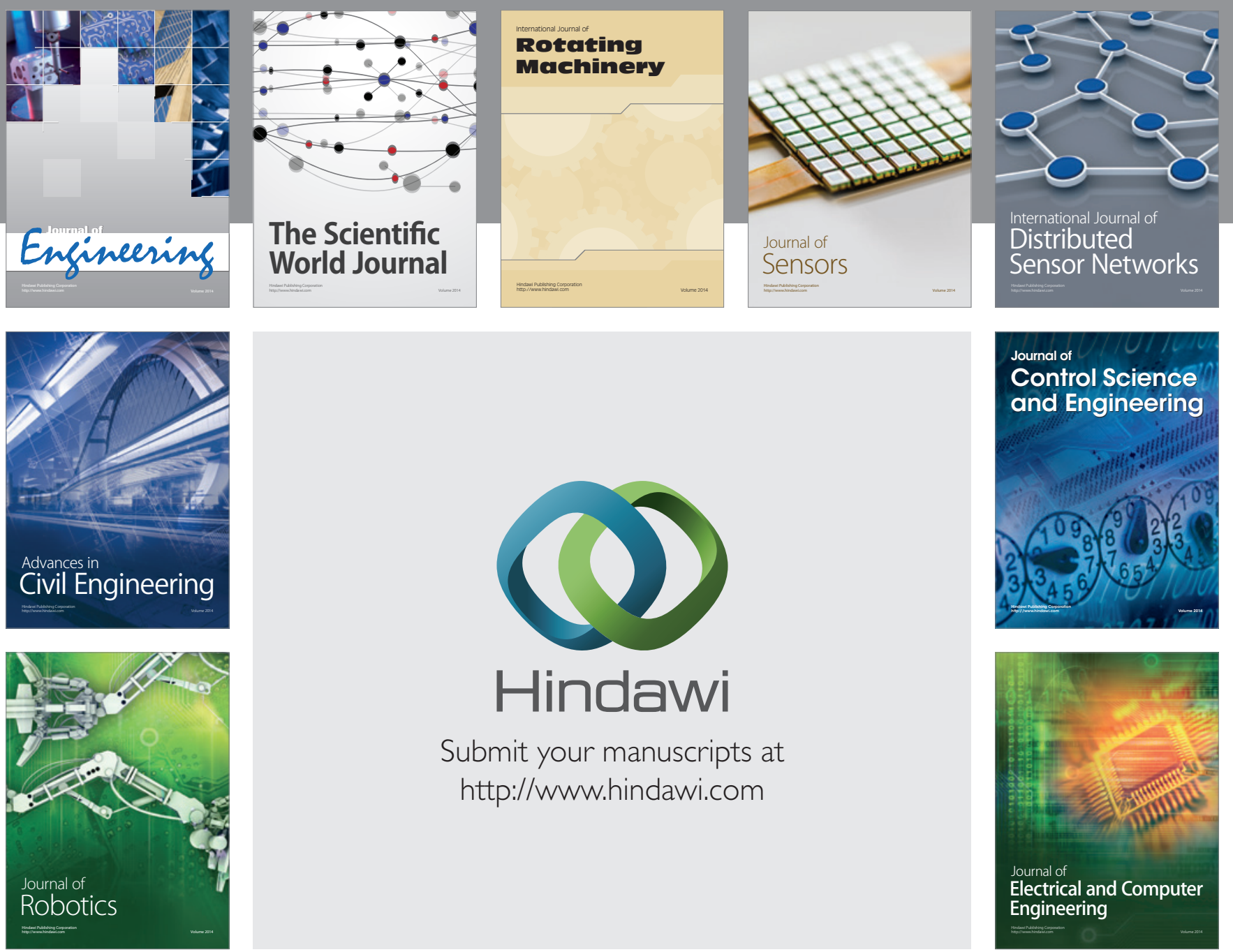

Submit your manuscripts at

http://www.hindawi.com
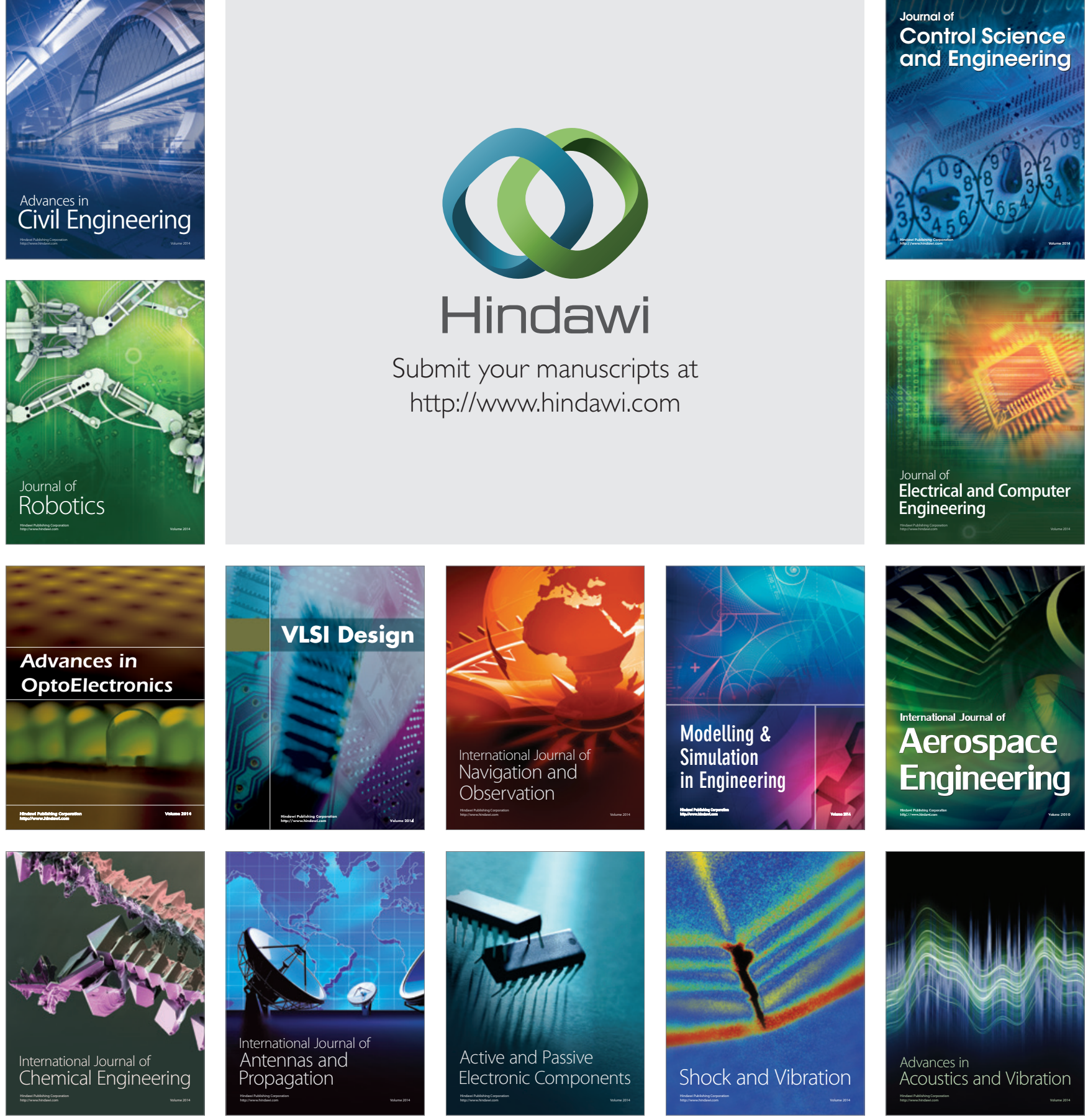